\title{
Twinlike Models for Self-Dual Maxwell-Higgs Theories
}

\author{
D. Bazeia ${ }^{1,2}$, E. da Hora ${ }^{1,3}$ and R. Menezes ${ }^{2,4}$. \\ ${ }^{1}$ Departamento de Física, Universidade Federal da Paraíba, 58051-900, João Pessoa, Paraíba, Brazil. \\ ${ }^{2}$ Departamento de Física, Universidade Federal de Campina Grande, 58109-970, Campina Grande, Paraíba, Brazil. \\ ${ }^{3}$ Department of Mathematical Sciences, Durham University, DH1 3LE, Durham, County Durham, U.K. \\ ${ }^{4}$ Departamento de Ciências Exatas, Universidade Federal da Paraíba, 58297-000, Rio Tinto, Paraíba, Brazil.
}

In this work we present a theoretical framework that allows for the existence of coherent twinlike models in the context of self-dual Maxwell-Higgs theories. We verify the consistence of this framework by using it to develop some twinlike self-dual Maxwell-Higgs models. We use a combination of theoretical and numerical techniques to show that these models exhibit the very same topological BPS structures, including their field configurations and total energy. The study shows that it is possible to develop a completely consistent prescription, which extends the idea of twinlike models to the case of vortices in Maxwell-Higgs theories.

PACS numbers: 11.10.Kk, 11.10.Lm

\section{INTRODUCTION}

Topological structures have been extensively used to study phenomena related to several areas of physics [1]. In particular, they are of direct interest to cosmology, since they can be formed in a rather natural way during phase transitions in the early universe. In the context of classical field theories, topological structures are described as finite energy solutions to some nonlinear field models. Usually, the models are endowed with a scalar potential which exhibits degenerate vacua, allowing for the spontaneous symmetry breaking mechanism. In this context, kinks [2], which are the simplest topologically non-trivial configurations, are the 1-dimensional solutions to a single real scalar field theory, while vortices [3] and monopoles [4] are, respectively, 2-dimensional solutions to some Abelian gauge theories and 3-dimensional solutions to non-Abelian gauge theories.

An interesting issue concerning the study of topological structures is that, in some particular cases, such structures, beyond solving the standard equations of motion, are also solutions to a set of coupled first-order differential equations, named Bogomol'nyi-Prasad-Sommerfield (BPS) equations [5]. In this context, since we do not need to deal with the second-order Euler-Lagrange equations of the model under investigation, the topologically nontrivial BPS configurations are easier to be obtained. Another advantage of studying BPS configurations is that they describe minimum energy solutions to the corresponding classical field theories.

Motivated by recent results on cosmology and superstring theories, a new type of classical field theories has been intensively investigated during the last years. These theories, named $k$-field models, are usually endowed with non-standard kinetic terms that change the dynamics of the model under investigation. In this context, topologically non-trivial BPS solutions may exist, and the features they engender can be quite different, or quite similar to the ones presented by their usual counterparts; see, for instance, Ref. [6].

In a recent work, Andrews, Lewandowski, Trodden and
Wesley noted that some k-theories can mimic the very same topological structures engendered by their standard counterparts, including their energy densities [7]. Since these models map each other solutions, they were named twinlike models. In another recent work, some of us studied the existence of twinlike models in the context of scalar field theories [8]. There, in particular, one used a combination of theoretical and numerical techniques in order to perform a detailed analysis of such models, including the presence of first-order differential equations and their applications to the braneworld scenario. Soon after, another work by Adam and Queiruga [9] made very good use of the first-order framework, to develop algebraic technique to construct twinlike theories. Yet more recently, another interesting result was introduced in Ref. 10], concerning the presence of twinlike models, where the models present not only the same solution with the same energy density, but also the very same stability features.

The above works on twinlike models deal with scalar fields. Thus, a rather natural question is how to construct consistent twinlike models under the action of gauge fields. In the present work we focus our attention on planar Maxwell-Higgs models and their topologically non-trivial self-dual solutions. To achieve this goal, we organize the work as follows: in the next section we introduce the generalized theory and we also present the theoretical framework which allows for the existence of twinlike self-dual Maxwell-Higgs models. In Sec. [II] we prove the consistency of such framework by using it to developed a few twinlike self-dual Maxwell-Higgs systems. Next, in Sec. IV] we perform a detailed numerical analysis of these systems and in Sec. V] we present conclusions and some perspectives concerning future investigations. 


\section{THE THEORETICAL FRAMEWORK}

Let us first present the model, which is defined by the planar Lagrange density

$$
\mathcal{L}=-\frac{h(|\phi|)}{4} F_{\mu \nu} F^{\mu \nu}+w(|\phi|)\left|D_{\mu} \phi\right|^{2}-V(|\phi|) .
$$

Here, $F_{\mu \nu}=\partial_{\mu} A_{\nu}-\partial_{\nu} A_{\mu}$ stands for the electromagnetic field strength tensor, $D_{\mu} \phi=\partial_{\mu} \phi+i e A_{\mu} \phi$ represents the usual covariant derivative and $V(|\phi|)$ is the spontaneous symmetry breaking potential which controls the scalar matter self-interaction. Also, $h(|\phi|)$ and $w(|\phi|)$ are dimensionless functions of the amplitude of the scalar field. We note that non-trivial choices to $h(|\phi|)$ and $w(|\phi|)$ will induce, respectively, non-standard dynamics to both the gauge and scalar fields. Also, there are interesting motivations concerning the use of these two functions and, in particular, the function $h(|\phi|)$ represents a generalized dielectric function, which can be used to describe interactions between quarks and gluons [11].

Since it is useful to deal with dimensionless fields, coordinates and parameters, let us introduce the mass scale $M$, which we use to implement the following scale transformations: $x^{\mu} \rightarrow x^{\mu} / M, \phi \rightarrow M^{\frac{1}{2}} \phi, A^{\mu} \rightarrow M^{\frac{1}{2}} A^{\mu}$, $e \rightarrow M^{\frac{1}{2}} e$ and $v \rightarrow M^{\frac{1}{2}} v$, where $v$ represents the spontaneous symmetry breaking parameter of the model under investigation. As a consequence, we get that $\mathcal{L} \rightarrow M^{3} \mathcal{L}_{g}$, where $\mathcal{L}_{g}$ stands for the dimensionless Lagrange density to be used from now on, which has the very same functional form of $\mathcal{L}$. Also, for simplicity, we choose $v=e=1$.

The standard procedure to search for the rotationally symmetric configurations is to consider such configurations as static solutions of the Euler-Lagrange equations of the model. In the present case, these equations are

$$
\begin{gathered}
\partial_{\mu}\left(h F^{\mu \nu}\right)=J^{\nu} \\
\partial_{\mu}\left(w \partial^{\mu}|\phi|\right)-A_{\mu} A^{\mu}|\phi| w=\frac{1}{2}\left|D_{\mu} \phi\right|^{2} \frac{d w}{d|\phi|} \\
-\frac{F^{2}}{8} \frac{d h}{d|\phi|}-\frac{1}{2} \frac{d V}{d|\phi|},
\end{gathered}
$$

where $F^{2}=F_{\mu \nu} F^{\mu \nu}$. Also, we take $J^{\mu}=-2 w|\phi|^{2} A^{\mu}$.

In the present case, the Gauss law for static fields can be written in the form ( $k$ represents spatial indices)

$$
\partial_{k}\left(h \partial^{k} A^{0}\right)=-2 w|\phi|^{2} A^{0}
$$

from which we note that such law is trivially satisfied by $A^{0}=0$ (temporal gauge). So, we fix this gauge and use it from now on.

We now look for static and rotationally symmetric configurations of the form

$$
\phi(r, \theta)=g(r) e^{i n \theta},
$$

$$
\mathbf{A}(r, \theta)=-\frac{\widehat{\theta}}{r}(a(r)-n),
$$

where $r$ and $\theta$ are the polar coordinates. Also, $n= \pm 1$, $\pm 2, \pm 3, \ldots$, represents the vorticity of the configuration. We use the above eqs. (5) and (6) into the Euler-Lagrange equations (2) and (3) to get

$$
\begin{gathered}
h\left(\frac{d^{2} a}{d r^{2}}-\frac{1}{r} \frac{d a}{d r}\right)+\frac{d a}{d r} \frac{d h}{d r}=2 w g^{2} a, \\
w\left(\frac{d^{2} g}{d r^{2}}+\frac{1}{r} \frac{d g}{d r}-\frac{a^{2} g}{r^{2}}\right)=\frac{1}{4}\left(\frac{1}{r} \frac{d a}{d r}\right)^{2} \frac{d h}{d g} \\
-\frac{1}{2}\left(\left(\frac{d g}{d r}\right)^{2}-\frac{g^{2} a^{2}}{r^{2}}\right) \frac{d w}{d g}+\frac{1}{2} \frac{d V}{d g} .
\end{gathered}
$$

These are the rotationally symmetric equations of motion for the fields $a(r)$ and $g(r)$, respectively.

In order to solve the Euler-Lagrange equations (7) and (8), we need to specify the model. In general, it can be done following the generalized model, i.e., choosing nontrivial functional forms to the functions $h(g)$ and $w(g)$. In this case, it is important to note that both functions must be positive-definite, in order to avoid problems with the positiveness of the total energy of the model; see the expression for the energy density below. We have to choose $h(g)$ and $w(g)$, and we also need to specify the potential $V(g)$ for the scalar matter self-interaction, which must allow for the spontaneous symmetry breaking mechanism.

Before specifying the model, let us recall that the main purpose of this work is to investigate the existence of coherent twinlike models in the context of classical Maxwell-Higgs field theories. In particular, we focus our attention on the study of twinlike BPS states, i.e., finite energy field configurations which can be described as static solutions of a set of first-order differential equations (the BPS equations) having the very same field profile and energy.

In this sense, it is instructive to note that the limit $w(g)=1$ leads the model (1) back to the model studied in Ref. [12]. Thus, the limit $h(g)=1$ leads us back to the standard Maxwell-Higgs electrodynamics. Thus, a very interesting choice to the symmetry breaking potential $V(g)$ for the scalar matter self-interaction is

$$
V_{s}(g)=\frac{1}{2}\left(g^{2}-1\right)^{2} .
$$

Now, according to the conventions stated above, the Euler-Lagrange equations of motion (7) and (8) for the rotationally symmetric solutions can be rewritten in the form

$$
\begin{gathered}
\frac{d^{2} a}{d r^{2}}-\frac{1}{r} \frac{d a}{d r}=2 g^{2} a, \\
\frac{d^{2} g}{d r^{2}}+\frac{1}{r} \frac{d g}{d r}-\frac{a^{2} g}{r^{2}}=g\left(g^{2}-1\right) .
\end{gathered}
$$


The equations (10) and (11) are solvable by solutions of the two first-order differential equations

$$
\begin{gathered}
\frac{d g}{d r}= \pm \frac{g a}{r}, \\
\frac{1}{r} \frac{d a}{d r}= \pm\left(g^{2}-1\right) .
\end{gathered}
$$

The static solutions $g(r)$ and $a(r)$ to the above first-order equations are the well-known Bogomol'nyiPrasad-Sommerfield (BPS) states related to the standard Maxwell-Higgs electrodynamics. These states solve the rotationally symmetric Euler-Lagrange equations of motion (10) and (11) by minimizing the total energy of the resulting solutions; see equations (22) and (23) below.

If we use non-trivial choices for the functions $h(g)$ and $w(g)$, the resulting Euler-Lagrange equations (7) and (8) will be much more sophisticated than the usual Eqs. (10) and (11). In this sense, it seems useful to calculate the general expression for the non-standard energy density, from which we can get interesting insights related to the non-usual BPS states. This expression is

$$
\varepsilon=\frac{h}{2}\left(\frac{1}{r} \frac{d a}{d r}\right)^{2}+w\left(\left(\frac{d g}{d r}\right)^{2}+\frac{a^{2} g^{2}}{r^{2}}\right)+V .
$$

Here we note that the presence of the functions $h(g)$ and $w(g)$ makes it very hard to obtain the BPS configurations related to the non-standard model (11). However, the existence of coherent twinlike BPS configurations, i.e., finite energy static solutions of the first-order eqs. (12) and (13), is still possible, and it is closely constrained by two important assumptions concerning the functions $h(g), w(g)$ and $V(g)$. The first one is the following differential relation

$$
w=h+\frac{\left(g^{2}-1\right)}{2 g} \frac{d h}{d g},
$$

which leads to a link between the functions $h(g)$ and $w(g)$. The second constraint is

$$
V(g)=h(g) V_{s}(g),
$$

which defines the potential $V(g)$ of the non-standard model (11) as a product between the function $h(g)$ and the self-dual potential (9) of the canonical Maxwell-Higgs model. As we demonstrate below, via the constraints (15) and (16), the non-standard energy functional (14) can be rewritten in a way such that the corresponding total energy is minimized by the first order equations (12) and (13). Furthermore, such minimum energy can be adjusted to mimic the very same value achieved by the standard BPS solutions. This adjustment can be done by tuning the boundary conditions to be imposed on $h(g)$, near the origin and asymptotically. We perform this tuning in the next Section.

Now, to search for twinlike BPS solutions to the firstorder equations (12) and (13), we need to know the boundary conditions to be imposed on the functions $g(r)$ and $a(r)$, near the origin and asymptotically. Near the origin, such functions must have no singularity. So, given the Ansatz (5) and (6), $g(r)$ and $a(r)$ have to behave according to

$$
g(r \rightarrow 0) \rightarrow 0 \quad \text { and } \quad a(r \rightarrow 0) \rightarrow n .
$$

Also, the twinlike BPS configurations must have finite total energy. In this case, as a condition to make the energy finite, the non-standard energy functional (14) must vanish for $r \rightarrow \infty$. So, asymptotically, the profile functions $g(r)$ and $a(r)$ must behave according to

$$
g(r \rightarrow \infty) \rightarrow 1 \quad \text { and } \quad a(r \rightarrow \infty) \rightarrow 0
$$

\section{TWINLIKE BPS MODELS}

Let us now focus our attention on the twinlike BPS configurations themselves. The main purpose here is to use the theoretical framework developed in the previous Section to assure the existence of such twinlike configurations. We start using (15) and (16) to rewrite the energy density (14) in a way such that the resulting total energy is minimized by the differential eqs. (12) and (13). The value achieved by such minimized energy depends on the behaviour of $h(g)$, near the origin and asymptotically. In this sense, by choosing appropriate boundary conditions, we tune this value to be the very same one gets in the context of the standard self-dual Maxwell-Higgs model (91).

In general, since the functions $h(g)$ and $w(g)$ are related to each other by the differential constraint (15), and the non-standard potential $V(g)$ is given by Eq. (16), the modified energy functional (14) can be rewritten in the form (see also Eq. (9))

$$
\begin{aligned}
\varepsilon= & \frac{h}{2}\left(\frac{1}{r} \frac{d a}{d r} \mp\left(g^{2}-1\right)\right)^{2}+w\left(\frac{d g}{d r} \mp \frac{g a}{r}\right)^{2} \\
& \pm \frac{1}{r} \frac{d}{d r}\left(\left(g^{2}-1\right) a h\right),
\end{aligned}
$$

from which we note that the corresponding total energy $E$ is minimized by the first order differential equations (12) and (13). In this case, the minimum energy is given by

$$
E_{b p s}=\int \varepsilon_{b p s} d^{2} r
$$

where

$$
\varepsilon_{b p s}= \pm \frac{1}{r} \frac{d}{d r}\left(\left(g^{2}-1\right) a h\right),
$$

is the minimum energy density, i.e., the energy density related to the first order equations (12) and (13). Also, Eq. (20) defines the Bogomol'nyi bound, i.e., the lower bound for the energy functional (14). 
We use Eq. (21) to see that the Bogomol'nyi bound (20) depends on the boundary conditions (17) and (18), and also on the boundary conditions to be imposed on $h(g)$ itself. Here, the interesting point is that there are infinitely many functional forms to $h(g)$ which obey the same general boundary conditions; as a consequence, there are infinitely many non-standard energy densities (all of them given by Eq. (21)) which achieve the very same Bogomol'nyi bound (given by Eq. (20)). Each one of these forms of $h(g)$ defines a different non-standard self-dual model. In this sense, since the first order equations (12) and (13), and also the boundary conditions (17) and (18), do not depend on $h(g)$, all these models exhibit the very same BPS solutions to the profile functions $g(r)$ and $a(r)$. Since these different models have the very same field configuration and total energy, we keep naming them twinlike models.

To better clarify how the theoretical framework developed in this paper works, let us first consider the standard Maxwell-Higgs model, which is defined by $h(g)=1$. In such context, due to the finite-energy boundary conditions (17) and (18), Eq. (20) gives the Bogomol'nyi bound (which is quantized according to the vorticity $n$ of the solutions)

$$
E_{b p s}=2 \pi|n|
$$

where

$$
\varepsilon_{b p s}=\mp \frac{1}{r} \frac{d a}{d r} \pm \frac{1}{r} \frac{d}{d r}\left(g^{2} a\right)
$$

in the minimum energy functional. Here, we point out the existence of infinitely many models (i.e., infinitely many functional forms to $h(g)$ ) which achieve the very same Bogomol'nyi bound (20). In fact, as the reader can easily check, any function $h(g)$ satisfying the boundary conditions ( $C$ is any non-negative constant)

$$
h(r \rightarrow 0) \rightarrow 1 \quad \text { and } \quad h(r \rightarrow \infty) \rightarrow C
$$

leads to $E_{b p s}=2 \pi|n|$.

In order to illustrate the above result, let us now consider some specific models. The first example, representing the first non-standard self-dual model, is defined by

$$
h(g)=g^{2}+1 \text {. }
$$

In this case, $C=2$. The energy functional (21) can be rewritten as

$$
\varepsilon_{b p s}=\mp \frac{1}{r} \frac{d a}{d r} \pm \frac{1}{r} \frac{d}{d r}\left(g^{4} a\right) .
$$

Then, via eqs. (20) and (26), we conclude that the modified model defined by (25) achieves the same Bogomol'nyi bound (22) achieved by the usual Maxwell-Higgs model.

The other non-standard self-dual model we present is defined by

$$
h(g)=\left(g^{2}+1\right)\left(g^{2}-1\right)^{2}
$$

with $C=0$ in this case. Here, the minimum energy density is

$$
\varepsilon_{\text {bps }}=\mp \frac{1}{r} \frac{d a}{d r} \pm \frac{1}{r} \frac{d}{d r}\left(a g^{2}\left(g^{6}-2 g^{4}+2\right)\right) .
$$

It is important to note that, due to the boundary conditions (17), (18) and (24), the second term in eqs. (23), (26) and (28) does not contribute to the integration process in (20). In this sense, we conclude that all the physical information concerning the Bogomol'nyi bound (22) achieved by the models previously presented is enclosed by the first term in eqs. (23), (26) and (28). So, from now on we refer to such first and second terms as the physical and the irrelevant ones, respectively. In fact, all models defined by a $h(g)$ of the form

$$
h(g)=1+G(g)
$$

exhibit a minimum energy functional given by

$$
\varepsilon_{b p s}=\mp \frac{1}{r} \frac{d a}{d r} \pm \frac{1}{r} \frac{d}{d r}\left(a g^{2}\left(1+\frac{g^{2}-1}{g^{2}} G\right)\right) .
$$

Then, as a consequence, such models possess the very same physical term, while exhibiting quite different irrelevant terms.

To end this Section, we point out that one can choose the boundary conditions (24) in a different way. Generically, one can define ( $N$ is any positive constant)

$$
h(r \rightarrow 0) \rightarrow N \quad \text { and } \quad h(r \rightarrow \infty) \rightarrow C .
$$

Here also, there are infinitely many functional forms to $h(g)$ which behave according to such boundary conditions. Each one of these forms defines a different nonstandard self-dual model. These models compose an infinity set of twinlike theories, since they exhibit the very same BPS configurations (given as solutions of the differential eqs. (12) and (13), according to the finite-energy boundary conditions (17) and (18)) and also achieve the very same Bogomol'nyi bound (given by Eq. (20)), which is

$$
E_{b p s}=2 \pi|n| N
$$

If we use a general $N$, none of the above models encloses the standard Maxwell-Higgs model, which is defined with $N=1$. We also note that the extended versions of (29) and (30) are quite obvious.

\section{TWINLIKE NUMERICAL SOLUTIONS}

Let us now perform the numerical study of the twinlike self-dual models presented in this work. We numerically solve the first-order differential equations (12) and (13), according to the finite energy boundary conditions (17) and (18). The resulting solutions are well-known, and can be found, for instance, in Ref. [1]. We then use such 
solutions to perform a detailed analysis of the energy of the vortices, including their minimum energy densities (23), (26) and (28). In this sense, we also depict, separately, the numerical solutions to the physical and to the irrelevant terms presented in the previous Section, from which we show (numerically) that the irrelevant terms do not contribute to the Bogomol'nyi bounds computed above. Some results are plotted below, for the case $n=1$.

In Fig. 1, we present the numerical solutions for the energy densities related to the twinlike BPS states, and we note that the solutions for (23) and (28) engender the same basic features: they reach their maximum values near the origin, and they decrease monotonically for all $r$. Here, it is interesting to note that the core of the twinlike solution (28) is smaller than that of the usual solution (23). On the other hand, we see that the solution for the minimum energy density (26) is quite different from the previous ones. In this case, such solution reaches its maximum value at some finite distance $R$ from the origin, and it is not monotonically decrescent for all values of the independent variable $r$. Finally, we point out that all the minimum energy densities previously depicted vanish asymptotically.

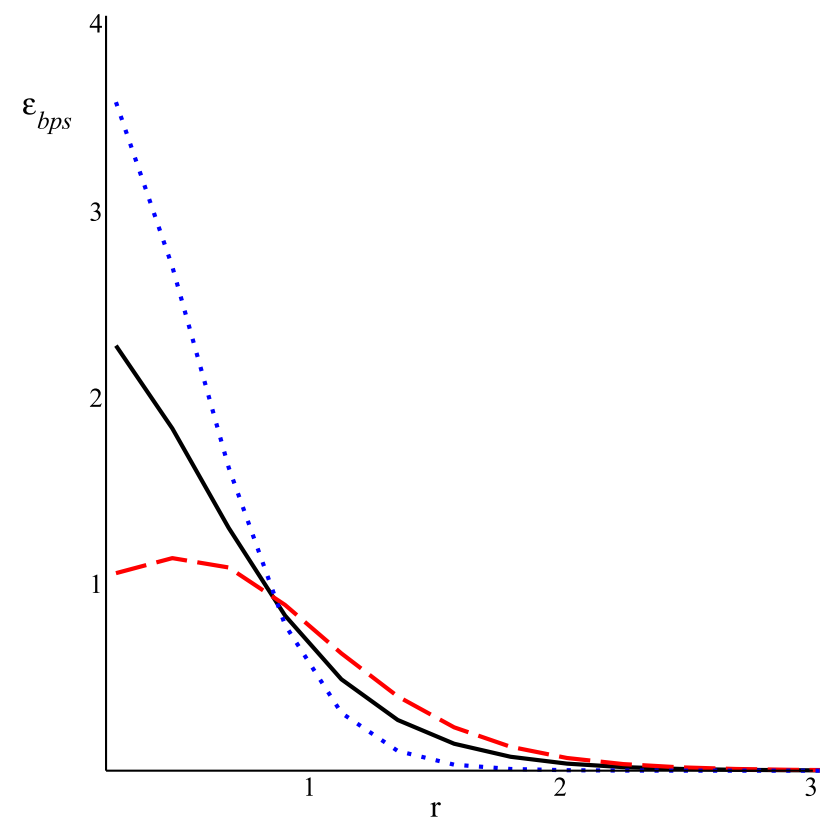

FIG. 1: Plots of solutions to the minimum energy densities (23) (black solid line), (26) (red dashed line) and (28) (blue dotted line).

In fact, since the minimum non-standard energy functional (21) is $r$-dependent only, the information regarding the Bogomol'nyi bound (20) is enclosed by the product $r \varepsilon_{b p s}$. In particular, the total energy $E_{b p s}$ of the twinlike BPS states is given by $2 \pi$ times the area enclosed by $r \varepsilon_{b p s}$. Regarding the non-standard models previously presented, such area is always equal to $|n|$. Thus, all the above models achieve the very same minimum total energy, which is given by the Bogomol'nyi bound (22).

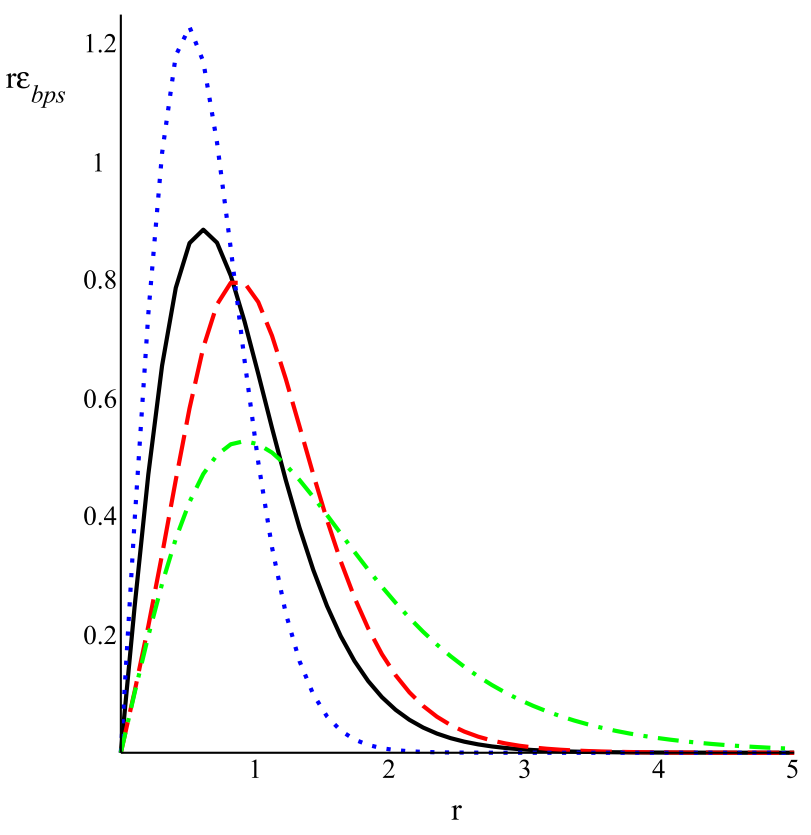

FIG. 2: Plots of solutions to the product $r \varepsilon_{b p s}$. Conventions as in FIG. 1. See also the solution to $-a^{\prime}$ (green dot-dashed line).

In Fig. 2, we depict the numerical solutions for the product $r \varepsilon_{\text {bps }}$ related to the previous models. In general, all the solutions engender the same features: they reach their maximum values at some finite distance from the origin, while vanishing for $r \rightarrow 0$ and for $r \rightarrow \infty$. It is interesting to note how these solutions behave in order to enclose the same area ever: there is an inverse relation between their maximum values and their characteristic lengths, and, as a consequence, the solutions with greater amplitudes exhibit smaller cores, and vice-versa.

In fact, the product $r \varepsilon_{b p s}$ means a summation over two different terms: the first one is given by $r$ times the physical term, and the second one is given by $r$ times the irrelevant term; see eqs.(23), (26) and (28). So, in order to get to some useful insights concerning the total energies of the twinlike BPS states, let us evaluate the numerical solutions for such terms, separately.

In the same Fig. 2, we plot the solution to $-a^{\prime}$ (prime means a derivative with respect to $r$ ). This solution is important since it denotes the product between $r$ and the physical term, and we note that it exhibits the same basic features the solutions to $r \varepsilon_{b p s}$ do, including the inverse relation between the amplitude and the characteristic length. Here, it is interesting to note that this solution also encloses an area equal to $|n|$. So, as stated theoretically before, our numerical analysis reinforces that all the physical information regarding the total energies of the non-standard models previously presented is enclosed by the physical term itself.

In Fig. 3, we depict the solutions to the second term, that is, the product between $r$ and the irrelevant term in the energy density. In this case, it is interesting to note 


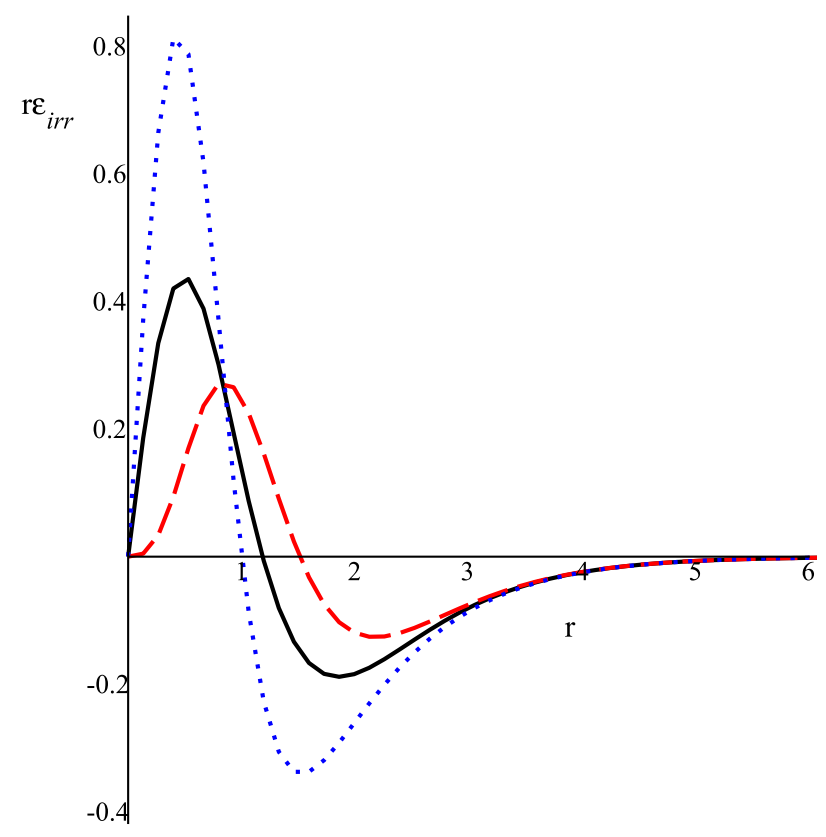

FIG. 3: Plots of solutions to the product between $r$ and the irrelevant terms. Conventions as in FIG. 1.

how these solutions behave in order to contribute nothing to the Bogomol'nyi bound. Here, we reinforce that, even in the presence of the negative sectors presented by such solutions (and, as a consequence, by the irrelevant terms themselves, since the radial coordinate $r$ is always positive), the positiveness of the minimum energy functionals (23), (26) and (28) is completely assured; see, for instance, the solutions depicted in Fig. 1.

To end this Section, let us investigate a very important issue regarding the study of topological configurations, which is the existence of conserved topological charges. In fact, the existence of such charges assures the topological stability of the corresponding configurations. In the present context, that is, for rotationally symmetric solutions of the form (5) and (6), the corresponding topological charge $Q_{T}$ can be identified with the flux of the magnetic field generated by such solutions themselves. So, one has

$$
Q_{T}=\int B d^{2} r \equiv \Phi_{B}
$$

where $\Phi_{B}$ stands for the magnetic flux, and

$$
B(r)=-\frac{1}{r} \frac{d a}{d r}
$$

is the magnetic field; see (6). According to our conventions, the magnetic flux (and, as a consequence, the topological charge) can be written as

$$
\Phi_{B}=2 \pi n,
$$

which shows that $Q_{T}$ is conserved, and is quantized according to the vorticity $n$. Since this result does not depend on $h(g)$, we think that the topological stability of the non-standard solutions is achieved in the same way as that of the standard case.

\section{ENDING COMMENTS}

In this work we investigated the existence of consistent twinlike self-dual classical field theories in the context of the planar Maxwell-Higgs model. We have used a modified Maxwell-Higgs model endowed by non-standard dynamics to both the gauge and the scalar fields. The unusual dynamics were introduced in terms of two nontrivial functions, $h(|\phi|)$ and $w(|\phi|)$, which are functions of the amplitude of the scalar field; they must be positive, in order to assure the positiveness of the energy functional of the modified model. We note that $h(|\phi|)$ is a kind of generalized dielectric function which couples to the canonical Maxwell action, leading to a non-standard dynamics to the gauge field. Also, $w(|\phi|)$ couples to the squared covariant derivative of the scalar field.

We have demonstrated the existence of twinlike selfdual Abelian-Higgs models under the presence of two specific constraints: the first one is a differential relation between the non-trivial functions $h(|\phi|)$ and $w(|\phi|)$, and the second one is a relation between $h(|\phi|)$ and the spontaneous symmetry breaking potential $V(|\phi|)$ of the non-standard model. We have used these constraints to performed a detailed numerical investigation, studying the energy spectra of the generalized models, including their minimum energy functionals. In general, we have noted that the numerical profiles for $\varepsilon_{b p s}$ itself can be quite different from one another, as they can engender different basic features. Even in this case, we have noticed that the solutions to $r \varepsilon_{b p s}$ engender the same features, including an interesting inverse relation between their maximum values and their characteristic lengths. In particular, such relation is very important, since it assures that all the solutions to $r \varepsilon_{b p s}$ enclose the same area, so all the non-standard BPS states have the very same total energy.

We hope that the above results may stimulate subsequent analysis in the field, mainly regarding the features that the twinlike Maxwell-Higgs models may engender. In particular, a rather natural issue concerns the extension of the present idea to the case of non-rotationally symmetric solutions. Another issue concerns the extension of the twinlike models to the context of Yang-MillsHiggs theories. These and other related issues are under investigation, and we hope to report on them in the near future.

The authors would like to thank CAPES, CNPq (Brazil) and FCT Project CERN/FP/116358/2010 (Portugal) for partial financial support. E. da Hora thanks the Department of Mathematical Sciences of Durham University (U.K.), for all their hospitality while doing this work. 
[1] A. Vilenkin and E. P. S. Shellard, Cosmic Strings and Other Topological Defects (Cambridge University Press, Cambridge, England, 1994); N. Manton and P. Sutcliffe, Topological Solitons (Cambridge University Press, Cambridge, England, 2004).

[2] D. Finkelstein, J. Math. Phys. 7, 1218 (1966).

[3] A. A. Abrikosov, Sov. Phys. JETP 5, 1174 (1957); H. B. Nielsen and P. Olesen, Nucl. Phys. B 61, 45 (1973).

[4] G. 't Hooft, Nucl. Phys. B 79, 276 (1974); A. M. Polyakov, JETP Lett. 20, 194 (1974).

[5] E. Bogomol'nyi, Sov. J. Nucl. Phys. 24, 449 (1976); M. Prasad and C. Sommerfield, Phys. Rev. Lett. 35, 760 (1975).

[6] E. Babichev, Phys. Rev. D 74, 085004 (2006); Phys. Rev. D 77, 065021 (2008); D. Bazeia, L. Losano, R. Menezes and J. C. R. E. Oliveira, Eur. Phys. J. C 51, 953 (2007); X. Jin, X. Li and D. Liu, Classical Quantum Gravity 24, 2773 (2007); C. Adam, N. Grandi, J. SanchezGuillen and A. Wereszczynski, J. Phys. A 41, 212004 (2008); Erratum-ibid. A 42, 159801 (2009); C. Adam, J. Sanchez-Guillen and A. Wereszczynski, J. Phys. A 40, 13625 (2007); Erratum-ibid. A. 42, 089801 (2009); C. Adam, N. Grandi, P. Klimas, J. Sanchez-Guillen and A.
Wereszczynski, J. Phys. A 41, 375401 (2008); C. Adam, P. Klimas, J. Sanchez-Guillen and A. Wereszczynski, J. Phys. A 42, 135401 (2009); D. Bazeia, E. da Hora, C. dos Santos and R. Menezes, Phys. Rev. D 81, 125014 (2010); D. Bazeia, E. da Hora, R. Menezes, H. P. de Oliveira and C. dos Santos, Phys. Rev. D 81, 125016 (2010); C. dos Santos and E. da Hora, Eur. Phys. J. C 70, 1145 (2010); Eur. Phys. J. C 71, 1519 (2011); C. dos Santos, Phys. Rev. D 82, 125009 (2010).

[7] M. Andrews, M. Lewandowski, M. Trodden and D. Wesley, Phys. Rev. D 82, 105006 (2010).

[8] D. Bazeia, J. D. Dantas, A. R. Gomes, L. Losano and R. Menezes, Phys. Rev. D 84, 045010 (2011).

[9] C. Adam and J. M. Queiruga, Phys. Rev. D 84, 105028 (2011).

[10] D. Bazeia and R. Menezes, Phys. Rev. D 84, 125018 (2011).

[11] R. Friedberg and T. D. Lee, Phys. Rev. D 15, 1694 (1977); Phys. Rev. D 16, 1096 (1977); Phys. Rev. D 18, 2623 (1978).

[12] D. Bazeia, Phys. Rev. D 46, 1879 (1992). 